\title{
Harrod sob análise: path-dependence, tempo histórico e mudança estrutural endógena
}

\author{
RICARDO RAMALHETE MOREIRA*
}

Harrod under analysis: path-dependence, historic time and endogenous structural change. The article aims to demonstrate how the Harrod's approach $(1937,1938,1948)$ can offer theoretical elements to form a complex, historicists and non-determinist view of the economic system. The relaxation of the constant warranty rate hypothesis make possible the system suffers endogenous qualitative change. It results in the notion of path-dependence and historic time. By the endogenization of the expectations and the existence of turn-points mechanisms, this approach allows a synthesis between non-convergency and economic regulation.

Keywords: Harrod; instability; regulation; path-dependence.

JEL Classification: B1; E3; O4.

\section{INTRODUÇÃO}

Harrod $(1937,1938,1948)$ tinha por objetivo tratar da dinâmica do objeto keynesiano de estudo. Keynes (1936) tratara da determinação da renda e do emprego, em termos estáticos ${ }^{1}$, não fazendo uso de taxas de crescimento. Keynes também mostrara a tendência do sistema capitalista operar abaixo do nível de pleno emprego, mostrando a necessidade da regulação estatal a fim de que o emprego fosse aproximado daquele nível. Harrod, por sua vez, estava preocupado

\footnotetext{
* Professor no Programa de Pós-Graduação em Economia da Universidade Federal do Espírito Santo (UFES). E-mail: ramalhete.s@gmail.com. Submetido: 14/11/2008; Aprovado: 28/5/2010.

${ }^{1}$ Embora a Teoria Geral de Keynes (1936) tenha elementos para uma análise dinâmica das economias monetárias, pode-se afirmar que, de forma geral, ao lidar com o tempo de produção (curto prazo), Keynes analisa a determinação da renda a partir de um modelo de equilíbrio estático (Kregel, 1976).
} 
com a dinâmica do sistema econômico; com sua taxa de crescimento ao longo do tempo e com a natureza dinamicamente instável do capitalismo².

Harrod busca mostrar que a definição de um conceito de crescimento garantido ou estacionário é fundamental no estudo da dinâmica econômica. Ele acreditava estar criando um instrumental teórico novo, capaz de lidar com fenômenos que envolvam processos dinâmicos, fazendo um contraponto ao instrumental neoclássico então vigente, este ligado ao estudo de fenômenos estáticos. A análise harrodiana, portanto, não está preocupada com niveis de produto ou emprego uma vez que apresenta caráter dinâmico -, mas com as taxas de expansão desses níveis. Duas são as questões básicas com as quais Harrod está empenhado, questões que serão estudadas ao longo deste artigo: i) Em primeiro plano, ele quer provar que o equilíbrio dinâmico é instável, ao contrário do que ocorre com o equilíbrio estático: ou seja, para Harrod, quando a taxa efetiva de expansão da economia torna-se diferente da taxa de equilíbrio (taxa garantida), essa diferença entre as duas se amplifica no tempo, ao invés de se reduzir. Segundo Kregel (1980), nesse primeiro momento, a taxa garantida (warranted rate) seria tratada por Harrod, para fins analíticos, como sendo constante ou dada; tratamento este que corresponderia à aplicação do modelo de equilíbrio estacionário utilizado por Keynes em sua Teoria Geral e mais bem identificado por Kregel (1976); ii) Em segundo plano, Harrod precisa lidar com o tempo histórico que permite modificações no próprio valor da taxa garantida (warranted rate), ou melhor, ele precisa relaxar a hipótese de uma taxa garantida constante no tempo. Como bom teórico, Harrod faz uso, num primeiro momento, de uma perspectiva menos complexa do sistema econômico, como forma de mediação analítica, para, num passo seguinte, tratar a dinâmica econômica num nível mais elevado de realismo e significado. Isto significa a inclusão do fenômeno de path-dependence e de modificações estruturais na análise harrodiana, mediante a aplicação do modelo de equilíbrio móvel (shiftting equilibrium).

O presente artigo tem como objetivo principal demonstrar como a abordagem harrodiana fornece elementos analíticos apropriados para uma concepção complexa e historicista ${ }^{3}$ do sistema econômico, na medida em que rejeita as noções reducionistas e neoclássicas de maximização e convergência, ao mesmo tempo em que permite a consideração das propriedades auto-organizadoras de um sistema complexo, mediante as noções de trajetória dependente (path-dependence) e tempo histórico. Defende-se a tese de que tais elementos permitem a emergência de uma sintese entre desequilíbrio e regulação econômica, desde que as expectativas de lucro

\footnotetext{
${ }^{2}$ Segundo Herscovici, "Harrod tentou construir um modelo dinâmico a partir dos elementos estáticos da Teoria Geral de Keynes" (Herscovici, 2002, p. 2)

${ }^{3}$ Uma abordagem historicista pode ser entendida como aquela que permite ao tempo histórico desempenhar um papel próprio sobre o sistema estudado, através das modificações qualitativas ou estruturais experimentadas por este último.
} 
no longo prazo sejam endogenizadas e que se considere a operação de certos amortecedores, os quais impedem que os movimentos na renda sejam lineares no tempo.

\section{EQUAÇÃO FUNDAMENTAL, CONCEITOS E IMPLICAÇÕES PRINCIPAIS: O MODELO CANÔNICO HARRODIANO}

Nos parágrafos seguintes, descreve-se o modelo básico de Roy Harrod (1937, 1938, 1948) e suas principais implicações dinâmicas. Conforme assegurou o próprio Harrod (1938, p. 254), o que se segue não deveria ser julgado exclusivamente pela validade e conveniência das equações propostas. Estas envolvem algo maior, um modo de "pensar dinamicamente" certos problemas econômicos.

A Equação Fundamental harrodiana corresponde ao casamento entre o princípio do acelerador e a teoria do multiplicador. De fato, segundo Besomi (1996, p. 8), Harrod seria considerado mais tarde o precursor dos modelos de interação acelerador/multiplicador. Seja G a taxa efetiva de crescimento da renda ou do produto, significando:

A) $G=\frac{y_{1}-y_{0}}{y_{0}}$.

Onde $y_{0}$ é o produto agregado no período 0 e $y_{1}$ o produto no período 1 . Seja ainda $G_{w}$ a taxa garantida de crescimento, entendida como aquela taxa que, se realizada, deixaria os empresários satisfeitos por terem produzido a quantidade correta (Harrod, 1938, p. 255). A taxa garantida não corresponde a um crescimento de pleno emprego, mas a um crescimento em que, segundo as expectativas dos empresários, o investimento efetivo torna-se igual ao investimento desejado, em cada período do tempo $\mathrm{p}^{4}$. As decisões individuais de produção, e principalmente de expansão ou contração da produção, são determinadas pelos resultados prévios observados pelos empresários. Um aumento nas receitas de vendas no período t, acima do que era previsto, estimula o empresário a elevar o incremento da produção no período t+1. Logo, $G_{w}$ representa uma taxa de expansão que garante uma satisfação geral das expectativas empresariais, o que implica manutenção da taxa global de crescimento. Assim, quando $G=G_{w}$, há manutenção das expectativas de longo prazo dos agentes e $G_{w}$ se mantém constante no tempo ${ }^{5}$.

\footnotetext{
${ }^{4}$ Ao mesmo tempo, a taxa garantida constitui-se numa idealização, numa situação na qual os objetivos empresariais são satisfeitos. Todavia, em uma economia em que as empresas buscam aumentar seu poder de mercado e as decisões são feitas descentralizadamente, há pouca chance de que a coletividade dos empresários consiga realizar suas expectativas e objetivos de lucro.

${ }^{5}$ Não obstante, Harrod (1948, p. 82) deixa claro que a realização de $G_{w}$ não significa uma satisfação equivalente de todos os agentes econômicos. Pode haver mesmo empresários insatisfeitos, alterando sua produção. Todavia, supõe-se que esta alteração seja contrabalançada por uma modificação de outro empresário, em sentido contrário, de maneira que, no agregado, a taxa de expansão seja mantida. Ou seja, $G_{w}$, de fato, oferece um crescimento que garante uma satisfação média das expectativas.
} 
Kregel (1980) define a taxa garantida com base na noção do modelo de equilíbrio estático utilizado na análise da Teoria Geral de Keynes. Deste modo, a taxa garantida, uma vez realizada $\left(G_{w}=\mathrm{G}\right)$, seria aquela que: a) manteria as expectativas de longo prazo constantes; b) permitiria uma realização das expectativas de curto prazo - expectativas não acerca de nível de renda, mas acerca de sua taxa de expansão; c) permitiria um estado de expectativas de longo prazo independente das expectativas de curto prazo. Seja $s$ a fração da renda que os agentes escolhem manter como poupança. Logo,

B) $s=S / y$

Sendo $S$ a poupança agregada (Besomi, 1996). Esta relação pode variar ao longo do ciclo econômico, embora ela seja considerada constante por Harrod, em um primeiro momento, para que seja verificada teoricamente a instabilidade do equilíbrio dinâmico. Todavia, sabe-se que, de fato, em momentos de recessão $s$ diminui; e cresce na expansão. Esses movimentos em $s$, historicamente determinados, explicariam, em princípio, a ocorrência de turning-points na teoria harrodiana. Chama-se $C$ o valor dos bens de investimento requerido pela produção de uma unidade adicional de produto, ou seja, trata-se do coeficiente requerido de capital. Assim, $C=I / \Delta y$ (Besomi, op. cit.). A Equação Fundamental em sua forma mais simples torna-se:

(I) $G_{w}=s / C$

Segundo Harrod, o valor da taxa garantida seria desconhecido e dependeria de certas "condições fundamentais", tais como a propensão a poupar, o estado tecnológico etc. A dinâmica do modelo, segundo o próprio Harrod, estaria neste desconhecimento do valor da taxa garantida de crescimento (Harrod, 1938, p. 256). Por outro lado:

(II) $G=s / C_{p}$

A taxa efetiva de crescimento $G$ é dada pela relação entre a propensão a pou$\operatorname{par}(s)$ e o coeficiente efetivo de capital $\left(C_{p}\right)$. O conceito de coeficiente de capital é baseado na ideia de que a produção corrente pode ser sustentada com o estoque de capital existente e que o incremento na produção, no longo prazo e dinamicamente, é sustentado com um incremento naquele estoque (Harrod, 1948, p. 82). Segundo Herscovici (2006), Harrod adotaria a hipótese de um coeficiente efetivo constante, o que seria a base do princípio da instabilidade, impedindo o processo de convergência encontrado na versão neoclássica do modelo de Solow (1956). No

Ademais, pode-se dizer que essa satisfação média dos empresários, que ocorre quando o produto cresce à taxa $G_{w}$, não equivale a uma maximização no sentido neoclássico (Ótimo de Pareto). Como dito, o crescimento à taxa $G_{w}$, quando realizado, não impede que algum agente isolado esteja insatisfeito, o que implica um estado subótimo. 
modelo de Harrod, a constância do coeficiente efetivo de capital seria uma consequência da hipótese de uma taxa de juros constante (Jones, 1979), uma vez que:

$$
\begin{aligned}
& r=\Delta Y / \Delta K \\
& C_{p}=\Delta K / \Delta Y \\
& C_{p}=1 / r
\end{aligned}
$$

As três equações acima dizem o seguinte: no equilíbrio, segundo a ótica neoclássica, a taxa de juros $(r)$ é igual à produtividade marginal do capital $(\Delta Y / \Delta K)$. Por outro lado, o coeficiente de capital nada mais é do que a variação do capital, dada uma variação da renda $(\Delta K / \Delta Y)$. Assim, o coeficiente de capital é o inverso da taxa de juros. Se a taxa de juros é constante, o coeficiente efetivo de capital também se torna constante. Neste caso, sob a hipótese de uma taxa de juros constante, o processo de convergência para o crescimento equilibrado estaria impedido, já que, na presença de um desequilíbrio entre o coeficiente efetivo e o requerido, o efetivo não poderia se ajustar a fim de que fosse eliminado o hiato existente. A suposta rigidez da taxa de juros em Harrod impede a ocorrência do princípio neoclássico da substitutibilidade dos fatores: como não existe uma perfeita flexibilidade da taxa de juros, que nada mais é do que o preço no mercado de capital, este último não consegue se equilibrar ${ }^{6}$. Dado que:

$$
C_{p}=\Delta \mathrm{K} / \Delta \mathrm{Y}=\mathrm{I} / \Delta \mathrm{Y}=\mathrm{f}[(\mathrm{e}-\mathrm{i}) / \Delta \mathrm{Y}]
$$

O coeficiente efetivo de capital $\left(C_{p}\right)$ depende, em última instância, da diferença entre a eficiência marginal do capital e a taxa de juros $(e-i)$. Nada garante que a eficiência marginal do capital (e) seja aquela que permita um nível de investimentos (I) que faça o coeficiente efetivo convergir para o coeficiente requerido, independentemente de se adotar ou não a hipótese de rigidez na taxa de juros.

Um pequeno esclarecimento é importante. Com o que foi dito no parágrafo acima não se quer estabelecer uma dependência necessária da eficiência marginal do capital em relação à intensidade do capital na produção (relação $\Delta \mathrm{K} / \Delta \mathrm{Y}$ ou coeficiente de capital). Com efeito, nas tradições clássicas à la Smith, a taxa de retorno do capital era associada à sua produtividade observada, que por sua vez era concebida como inversamente relacionada com a intensidade capitalística de

\footnotetext{
${ }^{6}$ A resposta de Solow (1956) à abordagem harrodiana seria justamente rejeitar a hipótese de qualquer tipo de rigidez nos preços de mercado, concebendo uma concorrência perfeita na economia, o que faz com que haja plena substitutibilidade dos fatores e flexibilidade do coeficiente de capital. Assim, existe, no modelo de Solow (1956), uma convergência para o crescimento equilibrado, sendo a taxa de juros, em última instância, o mecanismo regulador do equilíbrio macroeconômico, tal qual na Teoria dos Fundos Emprestáveis. Harrod, contudo, adota convenientemente a hipótese de uma taxa de juros exógena, fator fundamental para que exista a instabilidade dinâmica em seu modelo canônico. Por outro lado, pode-se dizer que, mantendo a coerência com Keynes, mesmo se fosse suposta a flexibilidade da taxa de juros, ainda assim o coeficiente de capital poderia ser constante, já que ele também depende da eficiência marginal do capital.
} 
produção, ou seja, com a razão K/Y na produção. Logo, quanto maior essa razão, menor seria a produtividade do capital e menor seria a procura por novos investimentos em formação de capital fixo. No entanto, como bem se sabe, Keynes (1936) seria responsável por romper com esta noção ${ }^{7}$, visto que para ele a chamada eficiência marginal do capital estaria relacionada com a renda esperada ao longo da vida útil do equipamento, não com a renda ou produtividade obtidas com base no capital já instalado. Assim, a intensidade capitalística de produção não teria influência necessária sobre a eficiência marginal do capital e nem sobre as novas decisões de investimentos, estas baseadas em expectativas de longo prazo. Nesse sentido, o que se disse no parágrafo acima foi o seguinte: dada a renda esperada pelos empresários, o preço de oferta dos bens de capital e a taxa monetária de juros, define-se a eficiência marginal do capital e a demanda por novos investimentos, que por sua vez será responsável pela formação de novo capital instalado $(\Delta K)$. Portanto, a relação de causalidade é da eficiência marginal do capital para a formação de capital, e não desta última para a primeira - como explica, ainda que em outros termos, Pasinetti (1997). Com base nas equações I e II acima, chega-se à seguinte identidade:

$$
\text { (III) } G_{w} \cdot C=G \cdot C_{p}=s
$$

Quando $C_{p}$ é igual a $C$, ou seja, quando o coeficiente efetivo de capital é equivalente ao coeficiente requerido, a taxa de crescimento observada tem sido justificada pelas circunstâncias. Em outras palavras, o investimento realizado é igual ao investimento desejado, não havendo variação inesperada de estoques. Assim, quando em (III) $C_{p}=C$, tem-se que $\mathrm{G}=G_{w}$, dada a taxa de poupança $s$. Como ressalta Harrod (1938, pp. 257-258), C é uma variável ex ante, pois se refere ao montante de bens de capital que os produtores produziriam se lhes fosse possível prever as condições futuras da economia, não havendo espaço para frustrações de expectativas. Todavia, na presença de incerteza, nada garante que $\mathrm{C}$ (ex ante) seja igual a $C_{p}$ (ex post).

A fim de que seja demonstrada a instabilidade do equilíbrio dinâmico harrodiano, faz-se uma análise dos possíveis desequilíbrios entre $\mathrm{G}$ e $G_{w}$. Caso a renda esteja crescendo acima da taxa garantida, ou seja, se $\mathrm{G}>G_{w}$, os empresários perceberão uma diminuição dos estoques e dificuldades em atender às encomendas: haverá um estímulo por maiores investimentos a fim de incrementar a capacidade de produção da economia, o que se expressa pelo fato de $C_{p}<C$. Trata-se do fun-

\footnotetext{
${ }^{7}$ Ainda que isto seja controverso: na realidade, há espaço para se supor que Keynes, em sua Teoria Geral, estaria supondo que a expansão dos investimentos, no longo prazo, seria um fator de redução da eficiência marginal do capital, mesmo que com expressiva defasagem temporal. Esta seria para Keynes uma das condições de estabilidade do sistema econômico, impedindo que as flutuações fossem explosivas. Nas palavras do autor: "Quando o investimento, em dado tipo de capital, aumenta durante certo período, a eficiência marginal do capital diminui à medida que o investimento aumenta, em parte, porque a renda prospectiva baixará conforme suba a oferta deste tipo de capital [...]" (KEYNES, 1936, pp. 115-116).
} 
cionamento implícito da interação do multiplicador com o acelerador, ou seja, para que haja um crescimento contínuo do produto, supõe-se que elevações no investimento aumentem a renda, que por sua vez implique novas elevações no investimento.

Segundo Herscovici (2002), na expansão os investimentos efetivos são menores que os desejados. Assim, aumentando os investimentos (não necessariamente em formação líquida de capital ${ }^{8}$ ), os empresários aceleram o crescimento efetivo, via o mecanismo de interação multiplicador/acelerador, permitindo uma ampliação da divergência entre $\mathrm{G}$ e $G_{w}$. Por sua vez, quanto maior for a diferença $\mathrm{G}-G_{w}>0$, tanto maior será o estímulo para a acumulação de capital, elevando a diferença $C_{p}-\mathrm{C}<0$. Isto está de acordo com a declaração de Harrod (1938, p. 263), segundo a qual existem forças centrífugas operando fora da linha de crescimento garantido, forças estas cuja magnitude varia conforme a distância entre $\mathrm{G} \mathrm{e} G_{w}{ }^{9}$.

Quando, ao contrário, o produto está crescendo abaixo da taxa garantida $\left(\mathrm{G}<G_{w}\right)$, o investimento efetivo está acima do desejado, o que pode ser expresso por $C_{p}^{w}>$ C. Existe um mecanismo de contração autorreforçador: os empresários têm seus estoques aumentados e há uma redução de novas encomendas. Na recessão, as empresas diminuem seus investimentos, esforçando-se por diminuir a formação de estoques, uma vez que se espera uma demanda futura mais fraca. Entretanto, os esforços para reduzir investimentos ampliam os efeitos recessivos, alargando a diferença $\mathrm{G}-G_{w}<0$. Quanto maior esta diferença, tanto maior a redução de investimentos, o que se expressa pelo aumento de $C_{p}-\mathrm{C}>0$.

A abordagem harrodiana empresta relevância especial ao papel das expectativas empresariais na determinação da dinâmica econômica. Relevância essencial é dada à frustração das expectativas. Tanto a expansão econômica quanto a recessão econômica são engendradas por uma frustração de expectativas empresariais, que se expressa na diferença entre as taxas efetiva e garantida de crescimento, assim como entre os coeficientes efetivo e requerido de capital. Há expansão porque o produto cresce a uma taxa observada (taxa efetiva) que supera a taxa esperada (taxa garantida). As expectativas são frustradas de uma forma positiva, ou seja, há uma surpresa positiva para os empresários que veem seus estoques caírem abaixo do que era planejado. Isto os encoraja a elevarem a produção e, eventualmente, o nível de investimentos em formação líquida de capital; inversamente, há recessão, visto que o produto cresce a uma taxa observada menor que a taxa esperada pelos

\footnotetext{
${ }^{8}$ Os investimentos podem ser elevados com aumento da produção para formação de estoques. Seja a identidade Investimento = Formação líquida de capital fixo + Estoques. Logo, pode-se aumentar Investimento sem alterar a formação líquida de capital, cuja mudança requer modificações nas expectativas a longo prazo.

${ }^{9}$ Destarte, até este ponto, pode-se afirmar que há um processo de causação cumulativa — para usar o termo empregado nos trabalhos de Kaldor (1972) e Setterfield $(1997,1998)$-, fazendo com que o equilíbrio dinâmico no modelo de Harrod, expresso pela taxa garantida, seja fortemente instável: os desequilíbrios iniciais podem ser amplificados com o tempo. Trata-se de uma análise dinâmica que empresta um evidente caráter instável ao sistema econômico.
} 
agentes. Aqui, as expectativas são frustradas de um modo negativo, ou melhor, existe uma surpresa negativa para o empresariado, o qual vê os estoques ficarem acima do montante planejado. Por sua vez, tal contexto encoraja os empresários a reduzirem a produção, assim como, possivelmente, diminuírem os recursos destinados à ampliação da capacidade produtiva.

\section{AS REVERSÕES DE FASE NO MODELO DE HARROD}

Segundo Kregel (1980), quando a taxa garantida de crescimento é considerada constante, mas existem divergências entre $G_{w}$ e G, tem-se um modelo de equilíbrio estacionário, segundo a tipologia usada em Kregel (1976). A frustração das expectativas ${ }^{10}$ de curto prazo induzem ajustamentos correntes, porém as expectativas de longo prazo não são modificadas, pelo que $G_{w}$ fica constante. Todavia, ao contrário do que ocorre com a análise de determinação da renda no modelo keynesiano ${ }^{11}$, no modelo de Harrod os ajustamentos correntes, sob a hipótese de expectativas de longo prazo constantes, fazem com que os desequilíbrios aumentem no tempo. Por outro lado, Kregel (1980) mostra que o relaxamento da hipótese de expectativas de longo prazo exógenas dá origem ao modelo de equilíbrio móvel, também na análise harrodiana. Ou seja, quando se permite que a frustração nas expectativas de curto prazo dê origem a modificações nas expectativas de longo prazo, cria-se espaço para alterações na taxa garantida de crescimento $\left(G_{w}\right)$. De fato, é a aplicação do modelo de equilíbrio móvel na análise harrodiana que permite a introdução, nesta última, do fenômeno de path-dependence - este último entendido como o fato de que os valores a longo período do sistema são determinados pelas ocorrências de curto prazo (Moreira e Herscovici, 2006).

Em um período de expansão, situação em que $G>G_{w}$, se for suposta uma constância em $G_{w}$, ocorrerá, como já analisado, um processo autorreforçador de crescimento. Todavia, de fato, ao longo do processo de ajuste de $\mathrm{G}$, a taxa garantida também sofre modificações. Em um primeiro instante, quando $G>G_{w}$, a condição para que haja uma reversão de fase, ou seja, a condição para que o sistema experimente posteriormente $G<G_{w}$, é que a taxa garantida cresça acima da taxa efetiva, dando origem, em certo ponto, a um processo contrário, de recessão. A priori, durante uma expansão, espera-se que a fração da renda que é poupada $(s)$ aumente, enquanto o coeficiente requerido de capital, $C$, diminua. Esta elevação

\footnotetext{
${ }^{10}$ Expectativas acerca da taxa de crescimento da renda, e não acerca de níveis de renda.

${ }^{11}$ Sob a hipótese de constância nas expectativas de longo prazo, o modelo keynesiano apresenta um processo de convergência para o equilíbrio. Diante dos desequilíbrios entre a demanda agregada e a oferta agregada, os ajustamentos realizados pelos agentes têm como resultado fazer com que o sistema caminhe na direção do ponto de equilíbrio, ou seja, da demanda efetiva. Como ressaltara Harrod (1938), isso seria uma propriedade de uma análise estática do sistema econômico; sua análise dinâmica teria de lidar com a instabilidade do equilíbrio - com a não convergência para o crescimento equilibrado.
} 
da poupança pode ser interpretada pelos argumentos keynesianos, segundo os quais a propensão marginal a consumir decresce com o aumento da renda; logo, a propensão marginal a poupar aumenta com a elevação da renda (Keynes, 1936).

Por outro lado, durante a expansão, também segundo os argumentos keynesianos, existe uma força endógena que faz diminuir a eficiência marginal do capital, devido a uma redução da renda esperada (Keynes, op. cit.). Isto contribui para uma redução de $C$. Portanto, com um aumento de $s$ e uma redução de $C$, na equação (I), a taxa garantida $G_{w}$ cresce. Entretanto, para que o estudo das condições de reversão de fases no ciclo seja completo, torna-se necessário dar à equação (I) uma forma mais geral, proposta por Harrod (1938, p. 263). Tem-se que:

(I.2) $G_{w}=\frac{s-k-K / y}{C}$

A diferença entre as equações (I) e (I.2) está na inclusão, nesta última, das variáveis $k$ e $K / y$. Existe uma parcela de investimentos que está diretamente relacionada ao nível de renda corrente. A divisão deste tipo de investimento pela renda corrente $(y)$ é dada por $k$. Por sua vez, há uma parcela de investimentos cuja execução não depende da renda corrente. São investimentos relacionados com expectativas de longo prazo e de natureza exógena. $K / y$, assim, representa a fração da renda corrente gasta neste último tipo de capital. Em (I.2), o coeficiente requerido de capital $(C)$ passa a representar aquele montante de investimento por unidade adicional de produto não contabilizado em $k$ e em $K / y$ (Harrod, op. cit., p. 263).

Voltando à análise do período de expansão, o que ocorre em $G_{w}$ ? A medida em que a renda efetiva se eleva, $y$ aumenta, fazendo com que tanto $k$ quanto a relação $K / y$ diminuam (lembrando que $k$ representa uma relação dos investimentos endógenos para com $y$ ). Como $s$ cresce e $C$ se reduz, o resultado final dessas forças será uma taxa garantida crescente, enquanto durar a expansão. Pode-se supor que, durante certo intervalo da expansão, o crescimento de G é superior ao crescimento de $G_{w}$, o que origina um processo de causação cumulativa. Assim, em um primeiro intervalo da expansão, tanto G quanto $G_{w}$ crescem, porém tem-se $\frac{d G}{d t}>\frac{d G_{w}}{d t}$ e a diferença $\mathrm{G}-G_{w}>0$ se amplia. Todavia, em um segundo intervalo, é a taxa garantida que cresce acima da taxa efetiva $\left(\frac{d G}{d t}<\frac{d G_{w}}{d t}\right)$, permitindo que, a partir de certo instante, o valor de $G_{w}$ torne-se superior ao valor de $G$ - o que constitui-se na condição para a reversão de fase. Neste sentido, há um processo não linear nas variações da taxa garantida, para uma mesma fase do ciclo econômico. No início de uma expansão, é possível falar em um tipo de inércia, que mantém a taxa garantida menos sensível ao crescimento da renda corrente. Entretanto, a partir de certo momento da expansão essa inércia perde terreno e a taxa garantida se torna mais sensível ao crescimento da renda.

Essa suposta inércia é determinada pelas forças residuais que as convenções da fase precedente do ciclo ainda exercem sobre os agentes na fase corrente do ciclo. Como no fim de uma recessão a taxa garantida está diminuindo aceleradamente, no início de uma expansão esta tendência de queda ainda tem influência sobre a taxa garantida, fazendo com que sua elevação seja freada. A partir de 
certo ponto, contudo, tais forças residuais perdem efeito e a taxa garantida cresce de forma acelerada. Em termos de expectativas empresariais, pode-se supor o seguinte processo: no fim de uma recessão, as expectativas são extremamente pessimistas e, de fato, é este pessimismo das expectativas que permite uma recuperação econômica, visto que só assim a taxa garantida cai abaixo da taxa efetiva. Essa recuperação, no início da expansão, ainda apresenta um estado de expectativas enfraquecido ${ }^{12}$.

Enquanto na expansão o valor de $\mathrm{G}$ é superior ao valor $\operatorname{de} G_{w}$, tem-se que a acumulação requerida é maior que a efetiva $\left(C_{p}<\mathrm{C}\right)$. Quando, finalmente, a taxa garantida se torna superior à taxa efetiva, então a acumulação requerida torna-se inferior à acumulação efetiva $\left(C_{p}>C\right)$. Dá-se então início à recessão. Por outro lado, a reversão de uma depressão econômica é algo um pouco mais complexa, uma vez que, nesta fase, a taxa efetiva $(G)$ se torna negativa. Assim, para que tenha lugar uma recuperação econômica, não basta que a taxa garantida $\left(G_{w}\right)$ seja reduzida: ela também precisa se tornar negativa e menor que a taxa efetiva.

Existe uma diferença entre $\mathrm{C}$ e $C_{p}$ fundamental nesta análise: enquanto $\mathrm{C}$ sempre deve ser tratado como positivo, tanto na expansão como na contração, $C_{p}$ pode adquirir valor negativo. Isto ocorre quando $G$ é negativo e o numerador da equação (I.2) é positivo. Como ressaltou Harrod (1938, pp. 266-267), durante certo intervalo da depressão, enquanto $\mathrm{G}$ é negativo e $G_{w}$ ainda é positivo, o valor de $C_{p}$ adquiri sinal negativo. Isto significa que junto com a redução da renda e do produto ( $G$ negativo), há uma acumulação positiva de capital, ampliando as forças contracionistas na economia, ou seja, ampliando a crise de superprodução e forçando novas reduções na renda e no produto.

Durante esse processo de depressão, a taxa garantida $\left(G_{w}\right)$ continua a diminuir até chegar a um valor negativo: então, quando $\mathrm{G}$ e $G_{w}$ são ambos negativos, $C_{p}$ adquire novamente valor positivo. Quando $C_{p}$ é positivo, isto significa que uma redução da renda, ao invés de gerar uma acumulação positiva de capital, vai gerar uma decumulação de capital (acumulação negativa). Embora isto seja uma condição para a recuperação, esta só terá lugar de fato quando o valor de $G_{w}$ for menor que o valor de $\mathrm{G}$, estando ambos negativos: por exemplo, quando $G_{w}$ for $-2 \%$ e $G$ for $-1 \%$.

Quando $G_{w}$ e G são ambos negativos, mas $G_{w}>$ G (por exemplo: $G_{w}=-1 \%$ e $\mathrm{G}=-2 \%$ ), o processo de depressão continua a ter lugar. Neste sentido, $G_{w}$ continua a diminuir, à medida em que $s$ se reduz, $k$ e $K / y$ aumentam e $C$ aumenta. A partir de certo ponto, a redução em $G_{w}$ passa a ser mais rápida que a redução em $G$, ou seja $\frac{d G}{d t}<\frac{d G_{w}}{d t}$, permitindo a reversão de fase, no momento em que $G_{w}$ cair

\footnotetext{
${ }^{12}$ Ainda que a taxa garantida tenha uma nova tendência de elevação, já que ela está menor que a taxa efetiva, ainda assim existem os resíduos de convenções e hábitos da recessão recente, que impedem um fortalecimento acelerado das expectativas acerca da taxa de crescimento do produto. Não obstante, em certo momento tal inércia deixa de atuar, e a tendência de elevação da taxa garantida na expansão econômica pode ser facilmente observada, uma vez que as expectativas empresariais são significativamente melhoradas.
} 
abaixo de $G$, estando ambas as taxas negativas. Neste momento, quando $G_{w}$ e $G$ são ambas negativas e $G_{w}<\mathrm{G}$ (por exemplo: $G_{w}=-4 \%$ e $\mathrm{G}=-3 \%$ ), abre-se espaço para a recuperação e $\mathrm{G}$, a taxa efetiva, passa a $\operatorname{crescer}^{13}$.

\section{A TAXA NATURAL DE CRESCIMENTO NO MODELO DE HARROD}

Além das possíveis divergências entre as taxas efetiva e garantida, existe um outro tipo de divergências a ser analisado. Para tanto, resta apresentar o que Harrod (1938, p. 264) chamaria de taxa natural de crescimento $\left(G_{n}\right)$. A taxa natural representa o máximo de crescimento da renda ou produto permitido pelo crescimento populacional, pela acumulação de capital, pelas melhorias tecnológicas e pelas preferências. Neste sentido, a taxa natural é a que possibilita um crescimento de pleno emprego, ou de inexistência de desemprego involuntário (Harrod, 1948, p. 87) ${ }^{14}$. Analisemos primeiro o seguinte caso: a taxa natural é superior às taxas efetiva e garantida, enquanto esta última está abaixo da efetiva. Logo, $G_{w}<\mathrm{G}<G_{n}$. Como visto, há uma tendência, em um primeiro momento, de expansão cumulativa, uma vez que a taxa efetiva é superior à garantida. Todavia, a taxa natural representa o teto de crescimento efetivo, acima do qual a taxa efetiva, a longo prazo, não pode ficar. À medida em que $\mathrm{G}$ se aproxima de $G_{n}$, há uma pressão crescente sobre os custos correntes, implicando elevação de preços, ou seja, em aumento da inflação. Paralelamente, existe o aumento de $G_{w}$, dado o incremento em $s$, a redução em $k$ e $K / y$ e a diminuição em $C$. Quanto mais próxima estiver $\mathrm{G}$ de $G_{n}$, tanto mais rápida será a elevação em $G_{w}$.

Por outro lado, caso a taxa garantida ultrapasse a taxa natural, haverá a nova situação em que $G_{w}>G_{n}>$ G. Como a taxa efetiva se torna inferior à taxa garantida, dá-se um processo de redução de G: o sistema sofre a reversão de fase. É interessante observar que, quando $G_{w}>G_{n}$, a economia sofre necessariamente uma redução do crescimento, uma vez que $G$, no longo prazo, não pode ser superior à $G_{n}$. Mesmo que a taxa efetiva seja igual à natural $\left(G=G_{n}\right)$, i.e., mesmo havendo crescimento de pleno emprego, ainda assim $G$ começará a se reduzir, pois a taxa garantida está acima de $G_{n}$ e, logo, $G_{w}>$ G. Desta forma, pode-se afirmar que, quando a taxa garantida ultrapassa a taxa natural, aumenta a probabilidade de uma recessão antes que a economia alcance o pleno emprego. Deve ser observado

\footnotetext{
${ }^{13}$ Destarte, mediante a aplicação do modelo de equilíbrio móvel na abordagem harrodiana, torna-se possível uma explicação historicista do ciclo econômico, com base em: i) as frustrações nas expectativas de curto prazo implicam alterações nas expectativas de longo prazo dos agentes; ii) essas alterações, por sua vez, modificam a taxa garantida de crescimento. Trata-se, em outras palavras, do reconhecimento do fenômeno de path-dependence no processo de crescimento do produto numa economia sujeita à incerteza e ao tempo histórico.

${ }^{14}$ Pode-se afirmar que essa taxa é definida exogenamente e representa a variável de fechamento do modelo de Harrod. Ou seja, a taxa natural é representativa de um dado contexto histórico e institucional vigente.
} 
que $G_{n}$ representa um valor máximo para o crescimento no longo prazo. Após uma depressão econômica, $G$ pode alcançar um valor maior que $G_{n}$ por um curto período de tempo ${ }^{15}$. Todavia, não é possível, no longo prazo, manter um crescimento da produção acima do permitido pelo crescimento populacional e pelas melhorias tecnológicas (Harrod, 1948, p. 87).

O caso ideal seria o de uma igualdade entre as taxas efetiva, garantida e natural ( $\left.G_{w}=G_{n}=\mathrm{G}\right)$. Neste caso, haveria um crescimento efetivo em que o investimento desejado é igual ao realizado, sendo este montante de investimentos o necessário ao pleno emprego dos fatores. Não obstante, esta situação ideal tem poucas chances de ocorrer, pois: i) a taxa garantida é formada com base nas expectativas de longo prazo dos agentes, as quais dificilmente são realizadas em um contexto de incerteza do tipo Keynes-Knight; ii) a taxa natural é considerada, por fins analíticos, como um dado exógeno. Todavia, modificações históricas e institucionais podem alterar a taxa natural, tornando mais complexa aquela igualdade.

\section{INSTABILIDADE, REGULAÇÃO E MODIFICAÇÕES QUALITATIVAS}

Como visto, Harrod $(1937,1938,1948)$ defende o princípio da instabilidade dinâmica mediante, num primeiro momento, a suposição de uma taxa garantida constante. Ele quer provar que o equilíbrio dinâmico é instável, ou seja, para Harrod, quando a taxa efetiva de expansão da economia torna-se diferente da taxa de equilíbrio (taxa garantida), essa diferença entre as duas se amplifica no tempo, ao invés de se reduzir. Todavia, caso essa suposição de uma taxa garantida constante seja aceita indefinidamente, não há meios para se explicar o processo cíclico da economia, assim como a forma pela qual ocorrem os pontos de inflexão de fases (turning-points). Haveria, isso sim, processos indefinidos de expansão ou recessão econômicas. A hipótese de uma causação cumulativa deveria ser levada ao extremo na análise da dinâmica econômica: as flutuações seriam explosivas. Contudo, num segundo momento, Harrod relaxa a hipótese de uma taxa garantida constante. De fato, essa taxa varia ao longo do tempo, segundo variações em seus termos explicativos $(s, k, C, K, y)$. Isto permite uma certa regulação econômica, à medida que existem pisos e tetos para as fases cíclicas de recessão e expansão na economia. Neste caso, emerge a propriedade auto-organizadora de um sistema complexo, tal qual o sistema econômico.

Por sua vez, o sistema econômico passa a ser sujeito ao fenômeno de path-dependence, uma vez que frustrações nas expectativas de curto prazo, representa-

\footnotetext{
${ }^{15}$ Isto é possível porque, após uma depressão econômica, há uma redução considerável na taxa natural de crescimento, visto que há diminuição do estoque de capital, do fluxo de inovações tecnológicas e do contingente populacional. Assim, logo após uma depressão, quando as forças de recuperação econômica começam a se acentuar, a taxa efetiva pode ultrapassar a taxa natural, o que se verifica tão somente num curto espaço de tempo.
} 
das pelas diferenças entre $G$ e $G w$, determinam as alterações nas expectativas de longo prazo, fazendo com que $G w$ se modifique no tempo. Em certo sentido, as alterações em $G w$ expressam a noção de tempo histórico na dinâmica econômica desenvolvida por Harrod, havendo o reconhecimento da ocorrência de modificações qualitativas endogenamente determinadas ao longo do tempo (Moreira, 2006; Moreira e Herscovici, 2006). Harrod, a exemplo de Keynes (1936), permite uma perspectiva probabilista e não determinista da economia. Ele não concebe uma trajetória necessária ou predeterminada para o sistema, mas concede um instrumental teórico que permite explicar as várias situações e fases às quais está sujeita uma economia monetária de produção.

Pode-se ainda adicionar que Harrod permite uma harmonia entre instabilidade e regulação. Devido às especificidades da taxa de juros, e à consequente rigidez do coeficiente de capital efetivo, o sistema econômico apresenta uma dinâmica de não convergência, ou seja, os mercados, sozinhos, não garantem que a taxa efetiva venha a convergir para a taxa garantida. Neste sentido, a abordagem harrodiana surge como contraponto à abordagem de Solow (1956), pilar dos modelos neoclássicos de crescimento, que veem os mercados como meios ideais de coordenação e regulação econômica, permitindo uma convergência necessária do sistema para o crescimento dito equilibrado, e, sobretudo, de pleno emprego.

A abordagem harrodiana, contudo, ao rejeitar a noção de convergência necessária; ao admitir um funcionamento imperfeito ou ineficaz dos mercados, rompe frontalmente com a tradição iniciada por Solow. Todavia, mesmo concebendo a economia como estando sujeita a uma dinâmica de divergência, flutuações explosivas são contornadas, visto que o tempo histórico e a trajetória dependente (path-dependence) do sistema fazem com que a própria taxa garantida sofra alterações, no sentido de amenizar seus desvios em relação à taxa efetiva, mecanismo este que caracteriza uma propriedade auto-organizadora do sistema econômico. Desta forma, embora não existindo espaço para as noções neoclássicas de maximização e ótimo social em Harrod (1937, 1938, 1948), sua abordagem visualiza a emergência de formas alternativas de regulação (ordem relativa ou histórica) longe da posição de equilíbrio. Trata-se, por assim dizer, de uma síntese entre regulação e não convergência, ou seja, em outras palavras, de um tipo de teoria da divergência regulada.

Se as possíveis diferenças entre as taxas efetiva e garantida fossem indefinidamente crescentes, de certa forma, estaríamos supondo um sistema sem mecanismos reguladores. As decisões dos agentes não seriam coerentes no longo prazo com as informações disponíveis (por exemplo: a despeito de um persistente aumento dos lucros no curto prazo os empresários mantêm inalterados os seus planos de investimento e as suas expectativas de lucro no longo prazo). No caso de uma taxa efetiva maior que a taxa garantida $\left(\mathrm{G}-G_{w}>0\right)$, como visto, tem-se uma situação em que há redução de estoques, e o investimento desejado é maior que o investimento efetivo. Como consequência de uma demanda corrente maior que a esperada, os lucros são maiores que aqueles que satisfazem os empresários no longo prazo, de forma que eles aumentam a produção, no sentido de ajustá-la ao aumento da demanda e de equilibrar os estoques. Todavia, devido à operação do multiplicador, 
esse aumento de produção faz com que haja novas elevações nos gastos dos agentes. Assim, no período seguinte o hiato entre a taxa efetiva e garantida se eleva.

Esse hiato crescente entre ambas as taxas se mantém uma vez que a taxa garantida é considerada constante: neste âmbito de análise, as frustrações (positivas, no caso da expansão) nas expectativas de curto prazo não alteram as expectativas de longo prazo (não há path-dependence) e os investimentos em formação de nova capacidade produtiva não sofrem mudanças ${ }^{16}$. Todavia, o sistema capitalista apresenta como uma de suas características estilizadas a de ter relativo equilíbrio entre capacidade produtiva e demanda efetiva no longo prazo. Como explicar tal característica, em termos do modelo de Harrod? Isto implica mostrar que as flutuações não são explosivas: que existem mecanismos de regulação (fora do equilíbrio), os quais são responsáveis pela alternância de fases recessivas e expansivas da renda.

No caso de uma taxa efetiva maior que a garantida ${ }^{17}$, ocorre uma expansão desse hiato apenas num primeiro momento. À medida que a demanda corrente se situa persistentemente acima da demanda esperada, e os estoques planejados são sistematicamente maiores que os estoques efetivos, os empresários alteram suas expectativas de longo prazo, no sentido de que passam a esperar ou desejar uma maior taxa de lucro ${ }^{18}$ (aumento de demanda) para o longo prazo, concomitantemente ao fato de que decidem por elevar seus investimentos em expansão da capacidade produtiva ${ }^{19}$. Isto se expressa, no modelo de Harrod, por um aumento da taxa garantida, permitindo uma redução de sua distância em relação à taxa efetiva.

Ao mesmo tempo, determinados mecanismos amortecedores entram em cena com o aumento da renda. Dentre esses mecanismos, pode-se mencionar a propensão marginal a poupar $(s)$, que se eleva com o aumento da renda. Isto contribui para uma desaceleração da taxa efetiva, ao mesmo tempo em que a taxa garantida cresce, pois, como dito, os empresários requerem uma taxa de lucro no longo prazo mais elevada como consequência da expansão que escolheram por fazer na capacidade produtiva e da observação de um lucro de curto prazo sistematicamente superior ao que eles desejavam, anteriormente, para o longo prazo.

\footnotetext{
${ }^{16}$ Mesmo que certos amortecedores, tal como a propensão marginal a poupar (que é crescente na renda), atuem no sentido de tentar frear a expansão, esta tende a persistir, visto que a capacidade produtiva se mantém inerte ante a expansão da demanda e os empresários continuam com as mesmas "ambições" em termos de lucro desejado no longo prazo (taxa garantida constante). Trata-se de uma situação hipotética em que os lucros no curto prazo se mantêm sistematicamente maiores que os lucros desejados no longo prazo, porém sem que tal fenômeno faça com que os empresários aumentem suas estimativas de lucro no longo prazo. Assim, eles expandem continuamente a produção, mas sem que alterem suas decisões no que respeita à expansão da capacidade produtiva. Ora, em tal situação, as flutuações são mesmo explosivas.

${ }^{17}$ Obviamente, a análise é válida para o caso recessivo.

${ }^{18}$ Nesta análise, considera-se constante o estado tecnológico, assim como a estrutura de custos e eventuais mark-ups de mercado. Logo, há uma relação direta entre utilização da capacidade produtiva e taxa de lucros.

${ }^{19} \mathrm{O}$ que pressupõe a endogenização das expectativas de longo prazo, ao menos na análise da dinâmica econômica de longo prazo.
} 
Ora, em certo momentum, a taxa efetiva, cujo aumento se desacelera, torna-se menor que a taxa garantida (pois esta sofreu suficientes elevações). Em outras palavras, as expectativas de longo prazo cresceram exacerbadamente (como efeito de uma persistente expansão da demanda), ao passo que a taxa efetiva - via mecanismos de inversão de tendência - não pôde manter seu vigor observado no passado. Embora Harrod (1948) não chegue a tal conclusão, é claro que os mesmos motivos que fazem com que a taxa garantida se eleve (quando $G-G_{w}>0$ ) são também os que fazem com que o crescimento da taxa efetiva se desacelere, até que haja uma inversão de tendência, ou seja, até que $\mathrm{G}-G_{w}<0$.

Uma modificação estrutural (ou qualitativa) endogenamente determinada surge quando existe uma alteração em um ou mais dos parâmetros estruturais do sistema estudado, a partir do comportamento das próprias variáveis relevantes no mesmo (Moreira e Herscovici, 2006). Ora, se considerarmos o estado de expectativas de longo prazo no modelo de Harrod como sendo um dos parâmetros estruturais na dinâmica econômica ${ }^{20}$, torna-se possível entender as mudanças na taxa garantida - que corresponde a determinado estado de expectativas de longo prazo - como caracterizando mudanças qualitativas endogenamente determinadas, visto que tais mudanças emergem como resultado da própria diferença entre a taxa garantida e a taxa efetiva de crescimento do produto. Neste caso,

IV) $G_{w^{t}}=f\left(G_{t-n}-G_{w^{t-n}}\right)$, onde $f^{\prime}>0$.

Em IV, mostra-se que a taxa garantida em t é função de uma diferença entre as taxas efetiva e garantida em $\mathrm{t}-\mathrm{n}$, sendo que quando essa diferença é positiva a taxa garantida está crescendo $\left(f^{\prime}>0\right)$. O termo $\mathrm{n}(\mathrm{em} \mathrm{t}-\mathrm{n})$ indica a ordem de períodos defasados que afetam o valor observado da taxa garantida em t. O Anexo do artigo apresenta uma especificação alternativa para as equações da taxa garantida e efetiva, algumas considerações sobre as limitações de formalização matemática no modelo canônico harrodiano e um breve experimento numérico.

A partir do exposto até este momento, pode-se fazer um corte conceitual entre

\footnotetext{
${ }^{20}$ A questão do que deve ser considerada a "estrutura" do sistema estudado é uma questão teórico-dependente, no sentido de que a estrutura será dependente dos parâmetros adotados, os quais, por sua vez, dependem do objeto de estudo de cada teoria. Uma teoria da determinação da renda, neste sentido, concebe uma dada estrutura, ao passo que uma teoria da distribuição concebe uma outra estrutura; assim também, outra é a estrutura concebida por uma teoria do crescimento. Destarte, ao contrário do que possa parecer, mudança estrutural não está ligada necessariamente a mudanças tecnológicas. Desde que uma teoria em questão consiga explicar como se dá uma mudança endógena em um ou mais parâmetros estruturais adotados, essa teoria passa a lidar com o fenômeno das mudanças estruturais endogenamente determinadas. No caso de Harrod aqui estudado, um dos parâmetros estruturais do sistema é o estado de expectativas de longo prazo, expresso pela taxa garantida. As mudanças endógenas nesta taxa, portanto, explicam as mudanças estruturais endógenas no sistema estudado. $\mathrm{O}$ estado da tecnologia é considerado dado. Trata-se de um outro parâmetro, cujas interações com as variáveis do sistema não são objeto de estudo neste trabalho. Logo, apesar de importantes os trabalhos desenvolvidos na linha de Pasinetti $(1981,1993)$, as mudanças técnicas e demais inovações tecnológicas não são elementos necessários para, no âmbito do que se pretende neste artigo, se explicar como ocorrem as mudanças estruturais do sistema.
} 
o que seria a dinâmica de curto prazo e a dinâmica de longo prazo, em termos dos elementos teóricos encontrados na abordagem harrodiana. A leitura que convencionalmente se faz da hipótese da instabilidade dinâmica harrodiana ${ }^{21}$ parece carecer, na literatura sobre o assunto, de maior profundidade analítica. Em geral, vê-se o modelo de Harrod (1948) como um modelo de flutuações explosivas, pelo que muitos o culpam de má especificação ou de erros teóricos significantes. Todavia, a leitura que aqui se apresenta parece oferecer um possível contorno a essas críticas feitas à abordagem harrodiana.

Vejamos: a hipótese da instabilidade dinâmica estaria restrita à dinâmica de curto prazo; este curto prazo, entendido como o tempo teórico em que não há mudanças endógenas nas expectativas de longo prazo, nem nos planos de investimento em capacidade produtiva por parte dos empresários. Neste caso, há sim uma expansão dos desvios entre taxas efetiva e garantida. Trata-se de enunciar as condições necessárias para a manutenção e cumulatividade das fases cíclicas da renda. Contudo, a análise da dinâmica de longo prazo oferece o estudo das condições que permitem a inversão entre essas fases do ciclo da renda. Assim, na dinâmica de longo prazo, a hipótese da taxa garantida constante é relaxada, uma vez que as expectativas de longo prazo são tidas como endogenamente determinadas e os investimentos em capacidade produtiva se tornam sensíveis em relação aos resultados observados. Em suma, a leitura que aqui se propõe do modelo de Harrod inclui as seguintes características conceituais: a) há path-dependence: as expectativas de longo prazo são determinadas pelas expectativas de curto prazo, decorrido um número suficiente de períodos; b) há complexidade: o sistema, mesmo fora de qualquer equilíbrio, cria formas de impedir desordens generalizadas (não há flutuações explosivas) - os desvios entre a taxa efetiva e garantida não são eliminados por um processo de convergência necessária, porém são amenizados por mudanças estruturais, as quais permitem uma alternância entre as fases expansiva e recessiva, ao longo da dinâmica de longo prazo; c) há tempo histórico: as mudanças na taxa garantida - que expressam modificações no estado de confiança no longo prazo - e a operação dos mecanismos amortecedores ocorrem dentro de um tempo histórico, ou seja, no âmbito de mudanças qualitativas. De certo modo, quando $G_{w^{t}} \neq G_{w^{j}}$ (sendo t e j dois períodos distintos quaisquer), houve, entre t e $\mathrm{j}$, uma mudança qualitativa (ou estrutural) endogenamente determinada.

\section{CONCLUSÕES}

A abordagem de Harrod $(1937,1938,1948)$ surge como contraponto em relação ao paradigma de Solow (1956), no que respeita ao processo de crescimento econômico: para Harrod, não existe um processo de convergência para o crescimento equilibrado, visto que as especificidades monetárias da taxa de juros, à la

\footnotetext{
${ }^{21}$ A hipótese de um desvio inicial que se amplifica indefinidamente no tempo.
} 
Keynes (1936), não permitem que os mercados sejam cleaned automaticamente. Ademais, como o investimento efetivo depende das expectativas de longo prazo dos agentes, mesmo supondo flexibilidade da taxa de juros, nada garante que as variações do coeficiente efetivo de capital sejam tais que o sistema venha a convergir para a trajetória de crescimento equilibrado.

Demonstrou-se como a abordagem harrodiana, ao evitar uma posição teórica reducionista, viabiliza uma síntese entre não convergência e regulação - o que representa a adoção da noção de tempo histórico e do caráter auto-organizador de um sistema complexo - à medida que as expectativas de lucro no longo prazo são endogenizadas e determinados amortecedores impedem que os movimentos na renda sejam lineares no tempo. Neste caso, a hipótese da instabilidade dinâmica fica restrita ao curto prazo. Na dinâmica de longo prazo, a endogeneidade das expectativas de longo prazo e a operação de amortecedores da renda impedem que as flutuações sejam explosivas: numa economia monetária, há uma alternância irredutível entre as fases recessivas e expansivas da renda e produto.

\section{REFERÊNCIAS BIBLIOGRÁFICAS}

BÉSOMI, D. (1996). “The making of Harod's dynamics”. Doctoral Thesis, Ph.D.Degree of Loughbourough University, October.

HANSEN, L. \& HECKMAN, J. (1996). "The empirical foundations of calibration”, Journal of Economic Perspectives, 10(1): 87-104.

HARROD, R. F. (1937). “Mr. Keynes and traditional theory”, in Robert Lekachman, ed., Keynes' General Theory: Reports of Three Decades, London: MacMillan \& Co Ltd.

HARROD, R. F. (1938). "An essay in dynamic theory: 1938 Draft”. Edited by Daniele Besomi. History of Political Economy, v. 28, n. 2, 1996: 253-280.

HARROD, R. F. (1948). Towards a Dynamic Theory. Some Recent Developments of Economic Theory and their Applications to Policy. London: MacMillan.

HERSCOVICI, A. (2002). "O modelo de instabilidade de Harrod: uma abordagem em termos de não linearidade”. Textos do Grupo de Estudos em Macroeconomia (GREM). Mimeo: Vitória.

HERSCOVICI, A. (2006). "O modelo de Harrod: natureza das expectativas de longo prazo, instabilidade e não-linearidade”. Economia e Sociedade, Campinas, v. 15, n. 1 (26): 29-55.

JONES, H.G. (1979). Modernas Teorias Do Crescimento Econômico: Uma Introdução. São Paulo: Atlas.

KALDOR, Nicholas (1972). "The irrelevance of equilibrium economics”. The Economic Journal, 82 (328): 1237-1255.

KEYNES, J. M. (1936). A Teoria Geral do Emprego, do Juro e da Moeda. São Paulo: Atlas, 1982. Primeira edição em inglês, 1936.

KREGEL, J. (1980). "Economic dynamics and the theory of steady growth: an historical essay on Harrod's 'knife-edge'”. History of Political Economy, 12 (1): 97-123

KREGEL, J. A. (1976). "Economic methodology in the face of uncertainty: the modelling methods of Keynes and the post-keynesians”. The Economic Journal, 86: 209-225.

MOREIRA, R. R. \& HERSCOVICI, A. (2006). "Path-dependence, expectativas e regulação econômica: elementos de análise a partir de uma perspectiva pós-keynesiana”. Revista de Economia Contemporânea, Rio de Janeiro, 10(3): 547-574.

MOREIRA, R. R. (2006). "Relativizando o dilema estabilidade versus instabilidade: Keynes, o mainstream e o conceito de bifurcação em Economia”. Revista Economia, 7 (1): 189-216. 
OREIRO, J. L. C. \& LEMOS, B. (2005). “Um modelo macrodinâmico pós-Keynesiano de simulação com progresso técnico endógeno". Economia (Campinas), v. 6, pp. 223-259.

PASINETTI, L. (1981). Structural Change and Economic Growth. Cambridge: Cambridge University Press.

PASINETTI, L. (1993). Structural economic dynamics. Cambridge: Cambridge University Press.

PASINETTI, L. (1997). “The marginal efficiency of investment”, in Harcourt, G e Riach, P.A. (orgs.). A 'Second Edition' of the General Theory. Londres: Routledge.

POSSAS, M. (1987). A Dinâmica da Economia Capitalista: Uma Abordagem Teórica, São Paulo: Brasiliense.

SETTERFIELD, M. (1997). "Should economists dispense with the notion of equilibrium?". Journal of Post Keynesian Economics, vol. 20 (1), pp. 47-76.

SETTERFIELD, M. (1998). "History versus equilibrium: Nicholas Kaldor on historical time and economic theory”. Cambridge Journal of Economics, vol. 22, pp. 521-537.

SIMONSEN, M.H. (1983). Dinâmica Macroeconômica. São Paulo: McGraw-Hill.

SOLOW, R. M. (1956). "A contribution to the theory of economic growth". Quarterly Journal of Economics, v. 70 (1): 65-94.

\section{ANEXO - UMA ESPECIFICAÇÃO ALTERNATIVA PARA AS TAXAS GARANTIDA E EFETIVA E UM BREVE EXPERIMENTO NUMÉRICO}

Como é ressaltado na literatura econômica, existem erros de formalização no modelo canônico proposto por Harrod (Possas, 1987). Uma das dificuldades encontradas para se compatibilizar as ideias harrodianas com a própria formalização fornecida por Harrod estaria na formalização estática de sua obra. Embora o autor estivesse tratando de uma temática fundamentalmente dinâmica, ou de um tema que deveria ser "pensado dinamicamente", a formalização do objeto de análise foi feita com base em equações não dinâmicas. No entanto, o presente trabalho abstrai as limitações de formalização e matematização nos trabalhos seminais de Harrod, acreditando, acima de tudo, na riqueza de ideias e de proposições do autor.

Por outro lado, a simples especificação das equações harrodianas básicas em termos de diferenças finitas não resolve necessariamente o citado problema de especificação ${ }^{22}$. Como se sabe, para que sejam observadas trajetórias econômicas complexas e não lineares em determinado experimento ou análise torna-se necessária uma especificação a partir de um instrumental matemático que explicite as características dinâmicas, evolutivas, regressivas e incertas das variáveis endógenas e dos parâmetros estruturais. Logo, equações lineares em diferenças finitas ou equações diferenciais lineares não cumprem necessariamente aquela tarefa. Muita vezes, o que se observa é a presença de um moto-perpétuo, em que a intensidade e a periodicidade das flutuações cíclicas são constantes no tempo, o que não caracteriza um dos fatos estilizados da economia capitalista, a saber a irregularidade do ciclo (Oreiro e Lemos, 2005).

A seguir, embora não seja o foco de análise, o presente trabalho oferece uma

${ }^{22}$ Uma formalização interessante está em Simonsen (1983). 
alternativa de especificação das equações para a taxa garantida e para a taxa efetiva, a fim de que seja testada numericamente sua consistência com as predições do pensamento harrodiano, objeto deste trabalho. Uma especificação possível para a função qualitativa IV seria:

$$
\text { V) } G_{w^{t}}=\alpha_{1} G_{w^{t-1}}+\alpha_{2} G_{w^{t-2}}+\ldots+\alpha_{n} G_{w^{t-n}}+\beta_{1}\left(\mathrm{G}_{\mathrm{t}-1}-G_{w^{t-1}}\right)+\ldots+\beta_{n}\left(\mathrm{G}_{\mathrm{t}-\mathrm{n}}-G_{w^{t-n}}\right)+\varepsilon_{t}
$$

Ora, a equação $\mathrm{V}$ quer dizer que a taxa garantida no período $t$ é determinada pelos seus valores defasados observados nos períodos $\mathrm{t}-1 \mathrm{a} \mathrm{t}-\mathrm{n}$, sendo os coeficientes $\alpha$ 's maiores que zero; ademais, a taxa garantida é determinada pelos desvios defasados entre a taxa efetiva e garantida nos períodos t-1 a t-n, sendo os parâmetros $\beta$ 's coeficientes positivos; e representa um choque aleatório com média zero e variância constante observado no período t.

Pode-se impor ainda uma condição de não linearidade na relação entre a taxa garantida em $t \mathrm{e}$ os desvios defasados entre a taxa efetiva e a taxa garantida. Como foi dito neste trabalho, durante um primeiro momento da fase cíclica, a taxa garantida é menos sensível aos desvios das taxas; há um tipo de inércia apresentada pela taxa garantida, de forma que os desvios se amplificam. No entanto, após arrefecidos os efeitos inerciais sobre a taxa garantida, esta responde mais rapidamente aos desvios, o que permite que em algum momento haja a reversão de fase cíclica. Esta não linearidade na velocidade da taxa garantida pode ser expressa por uma alteração dos parâmetros $\beta$ 's na equação V. Valores maiores desses parâmetros definem uma maior velocidade com que a taxa garantida responde a desvios observados. Assim, sejam as seguintes condições:

V.1) $\beta_{1}=\omega_{1}$ se $\left|G_{\mathrm{t}-2}-G_{w}\right|-2 \mid<\gamma ; \ldots ; \beta_{\mathrm{n}}=\omega_{\mathrm{n}}$ se $\left|\mathrm{G}_{\mathrm{t}-\mathrm{n}-1}-G_{w^{t-n-1}}\right|<\gamma ;$

V.2) $\beta_{1}=\eta_{1}$ se $\left|G_{\mathrm{t}-2}-G_{w}{ }_{t-2}\right| \geq \gamma ; \ldots ; \beta_{\mathrm{n}}=\eta_{\mathrm{n}} \mathrm{se}\left|\mathrm{G}_{\mathrm{t}-\mathrm{n}-1}-G_{w^{t-n-1}}\right| \geq \gamma ;$

V.3) Seja ainda $\omega_{1}<\eta_{1 ;} \omega_{2}<\eta_{2 ; \ldots ;} \omega_{\mathrm{n}}<\eta_{\mathrm{n}}$.

Ora, as três condições acima impõem um limite de desvio g a partir do qual há uma mudança nos parâmetros $\beta$ 's que definem a velocidade de ajustamento da taxa garantida. Assim, se no período t-3, por exemplo, o desvio absoluto (em módulo) de crescimento tiver sido superior ou igual ao valor de g, o parâmetro $\beta_{2}$, no período $t$, apresentará o valor $\eta_{2}$, que é superior ao valor $\omega 2$, elevando-se a velocidade da taxa garantida em t face aos desvios de t-2; o mesmo ocorre com todos os demais parâmetros $\beta$ 's na equação.

De fato, a equação $\mathrm{V}$ em conjunto com as condições acima tenta se aproximar de um comportamento não linear e complexo de ajustamento do sistema econômico ante desvios entre as taxas de crescimento. Por se tratar de uma equação dinâmica autorregressiva de ordem n, não linear e estocástica, sua solução requer uma simulação numérica. Pode-se ainda especificar a seguinte equação de determinação da taxa efetiva de crescimento, apenas para efeito de uma simulação computacional:

VI) $\mathrm{G}_{\mathrm{t}}=\phi_{1} \mathrm{G}_{\mathrm{t}-1}+\phi_{2}\left(\mathrm{G}_{\mathrm{t}-1}-G_{w}\right)=\left(\phi_{1}+\phi_{2}\right) \mathrm{G}_{\mathrm{t}-1}-\phi_{2} G_{w} t-1+\varphi_{\mathrm{t}}$ 
Sendo $\phi_{1}$ e $\phi_{2}$ parâmetros maiores que zero, a taxa efetiva em $t$ depende de seu valor em $\mathrm{t}-1$, dado o coeficiente de inércia, e do desvio observado em $\mathrm{t}-1$; seja $\phi_{\mathrm{t}}$ um choque de taxa efetiva de crescimento em t.

Com base na equação V, condições V.1 a V.3 e equação VI, faz-se uma breve simulação computacional ${ }^{23}$ a fim de que sejam observadas as trajetórias dinâmicas da taxa efetiva e garantida, assim como do desvio entre ambas, e seja verificada a eventual consistência dessas equações com algumas das predições que se podem retirar do pensamento de Harrod e ressaltadas pelo presente trabalho.

$\mathrm{Na}$ tabela abaixo são apresentados os valores das condições iniciais, dos parâmetros e dos choques impostos sobre o experimento numérico. Impõe-se sobre a simulação choques de taxa efetiva de crescimento $(\varphi)$ nos períodos $t+6, t+20, t+29$ e $\mathrm{t}+42$ nos valores de, respectivamente, $0,05,-0,03,0,08$ e $-0,04$. Por simplificação, adota-se valores iguais para $\eta_{1}, \eta_{2}$ e $\eta_{3}$, tal que $\eta_{1}=\eta_{2}=\eta_{3}=0,9$. O limite de desvio a partir do qual os parâmetros $\beta$ 's sofrem modificação é dado por $\gamma=0,05$.

\begin{tabular}{cccccc}
\hline \multicolumn{6}{c}{ Condições iniciais, parâmetros e choques } \\
\hline$\alpha_{1}$ & $\alpha_{2}$ & $\alpha_{3}$ & $\omega_{1}$ & $\omega_{2}$ & $\omega_{3}$ \\
0,3 & 0,2 & 0,1 & 0,5 & 0,4 & 0,3 \\
$\gamma$ & $\eta_{1}$ & $\eta_{2}$ & $\eta_{3}$ & $\phi_{1}$ & $\phi_{2}$ \\
0,05 & 0,9 & 0,9 & 0,9 & 0,5 & 0,5 \\
$\varphi_{\mathrm{t}+6}$ & $\varphi_{\mathrm{t}+20}$ & $\varphi_{\mathrm{t}+29}$ & $\varphi_{\mathrm{t}+42}$ & $\mathrm{G}_{\mathrm{t}-1}$ & $\mathrm{G}_{\mathrm{wt}-1}$ \\
0,05 & $-0,03$ & 0,08 & $-0,04$ & 0,05 & 0,05 \\
\hline
\end{tabular}

As trajetórias dinâmicas das taxas de crescimento garantida e efetiva, assim como dos desvios entre ambas, são apresentadas no gráfico abaixo, para uma simulação de 49 períodos. As seguintes características são observadas:

a) Observam-se flutuações irregulares, porém não explosivas nas taxas de crescimento e nos desvios entre elas. Esta seria uma característica tida como um fato estilizado das economias capitalistas (Oreiro e Lemos, 2005) e consistente com a noção de dinâmica complexa que poderia ser encontrada no pensamento harrodiano;

b) Neste sentido, a instabilidade dinâmica preconizada por Harrod é relativi-

\footnotetext{
${ }^{23}$ A metodologia de simulação baseia-se na ideia de calibração, ou seja, de se adotar valores para as condições iniciais e parâmetros do modelo com o intuito de se testar sua compatibilidade com certos fatos estilizados (Hansen e Heckman, 1996). No entanto, aqui a ideia de fatos estilizados é substituída pela ideia de predições teóricas. Ou seja, não se trata de avaliar se as trajetórias simuladas são consistentes com as evidências empíricas disponíveis, mas se aquelas são consistentes com as predições da teoria. Neste caso, as predições do pensamento harrodiano.
} 
zada, ou seja, trata-se de uma instabilidade verificada dentro de certos intervalos de tempo;

c) As mudanças estruturais refletidas na não linearidade dos parâmetros, ao lado do comportamento regressivo e dos choques (ou inovações) no tempo, fazem com que não seja possível prever com exatidão a amplitude e a intensidade das fases cíclicas; a única coisa que se pode esperar é que em algum momento haja uma inversão de fase (Herscovici, 2002);

d) Para a geração da dinâmica irregular verificada, não importa apenas a forma com que as taxas respondem - regressiva e não linearmente - aos seus próprios desvios e flutuações, mas também o componente de inovação ou incerteza, representado pelos choques impostos sobre a economia.

Dinâmica da taxa garantida, taxa efetiva e do desvio

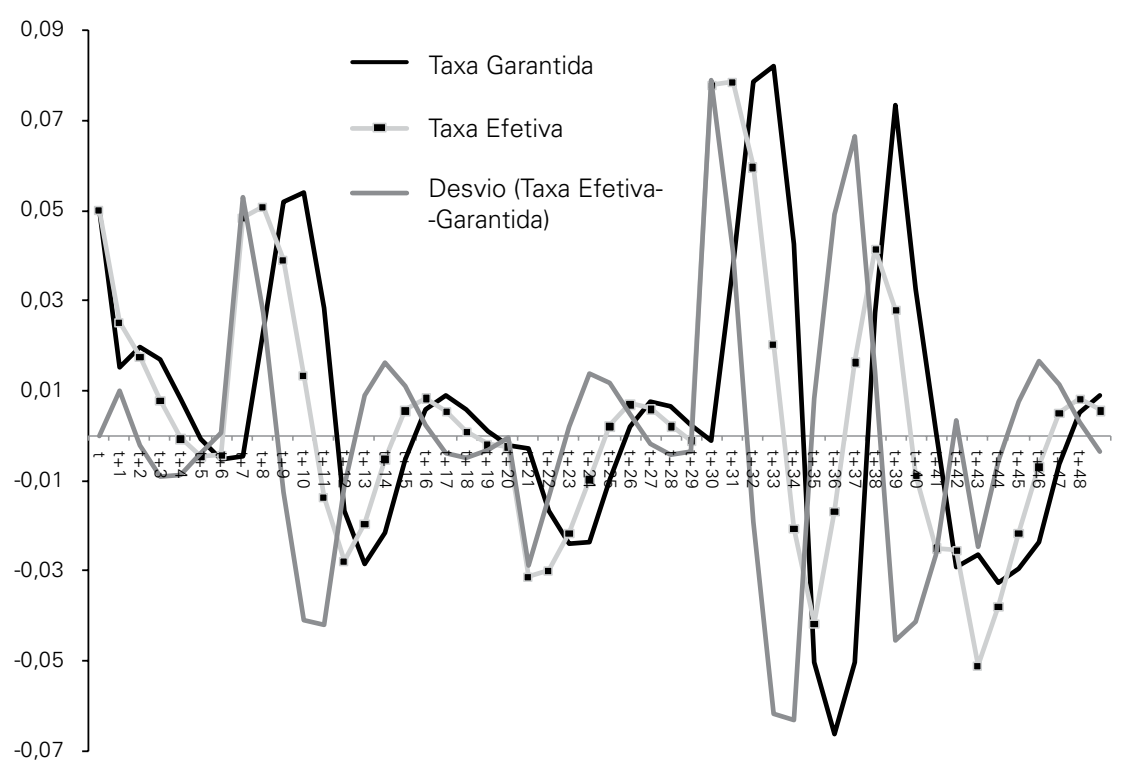

